\title{
st \\ El efecto Hall cuántico y sus contextos
}

Víctor Rodríguez

\begin{abstract}
$\stackrel{\sim}{\dddot{2}}$
RESUMEN

En este artículo, se atienden ciertas facetas conceptuales y experimentales del efecto Hall cuántico. Se argumenta que el mismo ofrece variados matices para la reflexión filosófica, desde la generación de entidades teóricas hasta la epistemología de la experimentación. La exposición pretende mantener cierta sensibilidad por la dinámica histórica en torno del tema, como así también por las implicaciones metrológicas de ámbitos cuánticos específicos. Dada la enorme producción científica sobre el tema, se hace un recorte a los fines de rescatar algunos perfiles significativos de los fenómenos asociados con ese efecto.
\end{abstract}

Palabras-CLAVe • Efecto Hall clásico. Efecto Hall cuántico. Topología cuántica.

\section{INTRODUCGIÓN}

Se analiza, en este artículo, un efecto físico que presenta matices interesantes para la reflexión filosófica en torno de la física cuántica. El abordaje que se realiza es próximo a la epistemología de la experimentación, interpretando que el sector experimental merece un tratamiento similar a otros más formales o más especulativos en el dominio de la filosofía de la física. Se atiende también a una dimensión histórica del fenómeno porque se considera que ella favorece la comprensión de las restricciones que suele producir la actividad experimental sobre los desarrollos teóricos. Adicionalmente, hay dos recortes importantes que deben mencionarse. Por un lado, prácticamente se han dejado de lado los tecnicismos propios de los enfoques teóricos actuales. Ello obligaría a extender el trabajo mucho más allá del límite propuesto para este artículo y en algunos tópicos ello está fuera de la competencia del autor. Se puede acceder a libros actualizados que se usan para dictar cursos sobre el tema, como Ezawa (2008), o Girvin (1999). Una excelente selección de artículos originales de gran valor teórico es Stone (1992). Por otra parte, no se considerarán desarrollos sumamente novedosos de los últimos años, tanto teórica como experimentalmente, que siguen llevando el nombre de efecto Hall, como el efecto Hall cuántico de espín. El motivo de esta decisión será comentado en la parte final del trabajo, por estimar que quedará más claro en esa fase de la exposición. 
El efecto Hall presenta un rostro clásico y varios rostros cuánticos. Se trata de un efecto que desde sus comienzos desafió a la ortodoxia. El efecto clásico surgió en el laboratorio como consecuencia de cierto escepticismo acerca de una reflexión de Maxwell y de las búsquedas para dar cuenta de una conjetura. El primer efecto cuántico apareció cien años después como una sorpresa, en un contexto de investigación de laboratorio orientado hacia otros objetivos. En pocos meses, cuando aún no se habían absorbido completamente las consecuencias de ese insospechado perfil cuántico, aparecieron otras manifestaciones más extrañas que mostraban la presencia de cargas fraccionarias en el ámbito de los electrones, algo nuevamente totalmente inesperado. A partir de allí, explotó un conjunto de líneas de investigación con una producción extraordinaria: un promedio de un artículo diario durante las últimas décadas. El contraste no puede ser mayor; un efecto clásico que fue tratado de modo secundario en los libros de referencia sobre electrodinámica clásica, ofrece en su versión cuántica inusitados perfiles que van desde nuevas entidades y propiedades, hasta la aparición de una constante que incide sobre el sistema metrológico internacional. Se sostiene que ese efecto merece un tratamiento al menos equivalente al que ha recibido la superconductividad y fenómenos relacionados en los últimos años dentro de la filosofía de la física.

Como es sabido, la filosofía de la mecánica cuántica ha crecido en varias direcciones. Sumada a la reflexión sobre numerosos temas de interpretación, la cuantización de los campos comenzó a apropiarse del derrotero conceptual en el área, y las partículas asociadas con ellos se transformaron en la cabeza visible o buscada de un gran iceberg. La producción filosófica en torno de la epistemología de la experimentación en ese terreno es considerablemente menor. Lo mismo sucede con algún sector de aplicaciones de la mecánica cuántica, como es el caso de la materia condensada. Sin embargo, esos campos de escalas intermedias están mostrando inusitadas propiedades que obligan a prestarle una debida atención. Un ingrediente central para una adecuada caracterización del contexto es la física de muy bajas temperaturas. Si comparamos ese sector con el de la física de los grandes aceleradores, también se trata de ámbitos de baja energía.

Las investigaciones están permitiendo comprender fenómenos relativamente complejos y, en particular, allanan algo el tratamiento siempre difícil de muchos cuerpos. Como es sabido, tales fenómenos escapan en primera instancia al alcance descriptivo de las formulaciones básicas de la teoría cuántica, las que usualmente funcionan bien en contextos de pocos elementos. Curiosamente, algunos de los nuevos ámbitos, usualmente tratados como híbridos, por las metodologías teóricas y experimentales usadas, están realizando aportes significativos de valor epistemológico. Hay temas en ese dominio de la física que exhiben ya una copiosa literatura, teñida de cuestiones 
epistemológicas cruzadas con aspectos conceptuales de la experimentación y con variadas elucubraciones en torno del concepto de modelo y sobre el arte de medir.

Una cuestión adicional que motiva este trabajo es el análisis de la complejidad y de la emergencia. Existe en varios ámbitos de la física una marcada tensión entre defensores de variantes del reduccionismo y propugnadores del emergentismo. Podemos tomar como ejemplos de esas posiciones a físicos como Weinberg (1993), por un lado, y Anderson (1972, 1995) o Laughlin (2005), por el otro. Aquí se intenta contemplar algún aspecto de esa tensión, pero relativamente desacoplada de los conceptos técnicos de complejidad y de sus múltiples rostros. Varios de ellos ameritan un tratamiento detallado en su vínculo con la física, pero lo que se pretende es solamente jerarquizar el ejemplo del efecto Hall como estudio de caso. Se intenta mostrar un terreno de ricas y complejas interacciones entre resultados experimentales no previstos, mediciones de alta precisión, y modelos provenientes de diferentes regiones de la física y de las matemáticas.

Atendiendo a las entidades teóricas, un concepto particularmente interesante íntimamente vinculado a investigaciones sobre el efecto Hall en los últimos años es el de cuasi-partícula. Cuando uno se aproxima al estudio de la física cuántica de comienzos de la década de 1930, se percata de que el vacío tiene propiedades especiales. Algunos fenómenos aparecen rotulados y, consecuentemente, entificados, como los agujeros de Dirac, y la predicción del positrón. Las excitaciones adquieren carta de ciudadanía, recordando en algún sentido a los viejos vórtices de Lord Kelvin. Es llamativo el modo de expresión similar, aunque con mucha más fuerza, que se encuentra en el contexto de las propiedades compuestas de los estados fermiónicos en trabajos recientes (cf. Jain, 2000). El dominio de algunas de esas cuasi-partículas sugiere una lectura fenomenológica en torno del concepto de ley física que hace difícil establecer una clara línea de distinción entre leyes y condiciones iniciales, una vieja y querida tradición en materia de investigación epistemológica.

El efecto Hall cuántico está conectado con esa temática. Si bien el fenómeno viene siendo conocido desde la segunda mitad del siglo xIx, llama la atención que hayan pasado cien años hasta que se descubrieran sus extrañas propiedades cuánticas. Los trabajos de von Klitzing, Störmer, Tsui, y Laughlin - cuatro premios Nobel por sus investigaciones sobre el efecto Hall -, han señalado el perfil del tratamiento actual del tema, pero los detalles teóricos han sido desarrollados posteriormente por numerosos autores, algunos de los cuales muestran una llamativa convergencia de estilos de investigación y notables condimentos de física especulativa que han tenido éxito experimental.

Una consideración adicional respecto de la tecnología. En este dominio se asiste a una interacción realmente sofisticada entre física y técnica, que abarca desde el diseño de experimentos, hasta las aplicaciones de resultados que se incorporan en nue- 
vas fases técnicas, en particular, en el tratamiento de los semiconductores. La física cuántica es un campo en el cual la filosofía se toca con la tecnología de una manera muy peculiar. No obstante, es conveniente recordar que, en esencia, el efecto en consideración aquí, surge de explorar relaciones entre el magnetismo y corrientes eléctricas bajo condiciones especiales. Ha sido la sensibilidad de los dispositivos lo que ha permitido observar los efectos cuánticos. Eso explica el paso del siglo mencionado. Hoy el campo aparece como una compleja red de tópicos interrelacionados que involucran aspectos de la física experimental, de la física teórica, de la física especulativa, de la matemática, de la tecnología y de la medición. A su vez, dentro de la física, además de la mecánica cuántica, el campo tiene relaciones con aspectos de la materia condensada, de las bajas temperaturas, de la teoría cuántica de campos, de la topología y, en especial, de lo que suele llamarse "materia exótica".

\section{LA EMERGENGIA DEL EFEGTO GLÁSIGo}

El año 1879 tiene valor simbólico para la historia de la física. Fue el año en que falleció James Clerk Maxwell y nació Albert Einstein. En particular, para los fines del presente trabajo, también fue el año en que Edwin Hall, publicó el artículo (1879). Este artículo comienza con una cita de un extenso párrafo de la primera edición del Tratado de electricidad y magnetismo (Treatise on electricity and magnetism) de Maxwell (1873).

Debe recordarse cuidadosamente que la fuerza mecánica que impulsa un conductor que lleva una corriente a través de las líneas de fuerza magnética, actúa, no sobre la corriente eléctrica, sino sobre el conductor que la lleva. Si el conductor es un disco rotatorio o un fluido se moverá obedeciendo a esa fuerza, y ese movimiento puede o no estar acompañado con un cambio de posición de la corriente eléctrica que lleva. Pero si la corriente misma es libre de elegir algún camino a través de un conductor sólido fijo o una red de alambres, entonces, cuando se hace actuar una fuerza magnética constante sobre el sistema, el camino de la corriente a través de los conductores no es alterado permanentemente, sino que luego de que ciertos fenómenos transitorios, llamados corrientes inducidas, se han apaciguado, se hallará que la distribución de la corriente es la misma que si no estuviera en acción la fuerza magnética. La única fuerza que actúa sobre las corrientes eléctricas es la fuerza electromotriz, la cual debe distinguirse de la fuerza mecánica que es el tópico de este capítulo. Maxwell (1873, v. 2, p. 144-5). ${ }^{\mathbf{1}}$ 
Hall reconoce que su motivación para intentar realizar el experimento, con lo cual explora la eventual validez de la observación de Maxwell, estuvo también influenciada por la lectura de otro artículo en el que se supone que un imán actúa sobre una corriente en un conductor fijo del mismo modo como actúa sobre el conductor mismo cuando se mueve libremente. Ante esos puntos de vista, decidió consultar al profesor Rowland, su supervisor de tesis, quien ya había intentado realizar algún experimento para chequear la consideración de Maxwell, aunque sin éxito. Estimulado por éste, Hall emprendió una serie de experimentos con un dispositivo que constaba básicamente de un alambre de plata germana en espiral plana entre dos discos delgados de caucho. El conjunto era colocado entre los polos de un electroimán, en posición tal que las líneas de fuerza magnética pasasen a través de la espiral en ángulos rectos a la corriente de electricidad. Un dato técnico importante es el rango del experimento. El alambre tenía un diámetro de alrededor de un milímetro y la resistencia era de aproximadamente dos ohms. La intensidad del campo magnético era posiblemente entre 15 . 000 y 20.000 veces la intensidad horizontal del magnetismo terrestre. El dispositivo le permitía detectar un cambio de alrededor de una parte en un millón en la resistencia de la espiral. Realizó 13 series de observaciones en pocos días, consistiendo cada una de ellas en 40 lecturas que alternaban la actividad del imán y un cambio en direcciones opuestas del mismo. No encontró cambios en la resistencia del rollo causados por la acción del imán. Esto lo llevó a ciertas reflexiones sobre la naturaleza de la electricidad y su comportamiento como fluido incompresible, considerando que era necesario chequear la diferencia de potencial entre puntos sobre los lados opuestos del conductor. Recurrió al diseño del experimento previo de Rowland con un disco de metal colocado entre los polos del electroimán, cruzando las líneas de fuerza. Colocó los dos polos del galvanómetro en diferentes partes del disco por el que pasaba una corriente eléctrica, hasta hallar dos puntos cercanamente equipotenciales. Activó la presencia magnética y observó el galvanómetro con la intención de detectar cualquier indicación de un cambio en el potencial relativo de los dos polos. El experimento dio resultado negativo y Hall lo atribuyó al espesor del disco. Aquí fue donde Rowland le sugirió usar láminas de oro montadas sobre una placa de vidrio. Con ese cambio aparentemente menor, tuvo éxito en detectar como efecto de la acción del imán una clara deflexión de la aguja del galvanómetro. Ésta era demasiado grande como para atribuírsela a la acción directa del imán sobre la aguja del galvanómetro, o alguna causa similar. Además, era permanente. El efecto se invertía cuando se invertía el imán. No se invertía cuando se transferían los polos del galvanómetro desde un extremo de la pieza al otro. Como dice Hall en su artículo, el fenómeno observado era justo lo que hubiera esperado ver, si la corriente eléctrica estuviera empujada, pero no movida, hacia un lado del conductor. Atendiendo a ese resultado y con la intención de realizar algún experimento cuantita- 
tivo, preparó un nuevo dispositivo para atender a las variaciones del efecto encontrado en función de variaciones de la fuerza magnética y la intensidad de la corriente a través de la hoja de oro. En un anexo al mismo artículo, Hall escribe que

Se puede quizás hablar de la acción del imán como estableciendo en la lámina de oro una nueva fuerza electromotriz a ángulos rectos de la fuerza primaria electromotriz. Esa nueva fuerza electromotriz no puede, bajo condiciones ordinarias, manifestarse por sí misma, si el circuito en el cual ella trabajara estuviera incompleto. Cuando se completa el circuito por medio del galvanómetro de Thomson, fluye una corriente. La corriente real a través del galvanómetro depende, por supuesto, de la resistencia del galvanómetro y de sus conexiones, tanto como de la distancia entre los dos puntos de la lámina de oro en los cuales se aplican los extremos de los alambres desde el galvanómetro (Hall, 1879, p. 292).

De ese modo, un aparente error de apreciación de Maxwell motivó la experimentación sobre un dominio de la electrodinámica clásica que condujo a un nuevo fenómeno. En la segunda edición del libro del Maxwell, el editor menciona brevemente el aporte de Hall. Lateralmente, es llamativa la astucia de Rowland acerca del tema, pero el efecto tiene merecidamente el nombre de su descubridor. El contexto general es difícil de captar plenamente desde una perspectiva conceptual actual, ya que hay que recordar que el electrón se descubrió recién 18 años después del artículo de Hall, y también que la fuerza de Lorentz, asociada a una carga puntual debida a campos electromagnéticos, con la que suele asociarse parte del fenómeno, no estaba desarrollada. Usualmente se atribuye esa fuerza a Heaviside, en un trabajo de 1889, y se interpreta que Lorentz la derivó poco tiempo después, en 1892.

Desde el punto de vista tecnológico, vale mencionar que se han generado numerosas aplicaciones a partir de ese efecto que han entrado en la tecnología electromagnética, aún de aplicación industrial. No se consideran aquí esas aplicaciones, pero es de señalar que como la magnitud y el signo del voltaje de Hall dependen de las propiedades de los materiales del conductor, se consigue una importante herramienta de diagnóstico para investigar los portadores de corriente eléctrica.

A pesar de esas aplicaciones, es adecuado llamar aquí la atención sobre el lugar insignificante que tuvo ese efecto en textos clásicos de electricidad y magnetismo. Dos ejemplos al respecto. En el libro de Panofsky y Phillips (1962), hay solamente una breve mención: "también despreciaremos un efecto adicional, conocido como el efecto Hall, que está presente aún a frecuencia cero, y da lugar a una redistribución de las superficies equipotenciales en un conductor portador de corriente. En todas, menos en las sustancia muy especiales, sin embargo, ese efecto es extremadamente pequeño" (p. 118). En el clásico libro de Jackson (1980), se lo ubica dentro de un problema. 
"Problema 6.16. Si en un conductor, o semiconductor, está fluyendo una corriente debida al campo eléctrico aplicado, y se aplica un campo magnético transversal, aparece una componente del campo eléctrico en la dirección ortogonal a ambos, el campo eléctrico aplicado (dirección de la corriente) y el campo magnético, lo que da lugar a una diferencia de potencial entre los lados del conductor. El fenómeno se conoce como efecto Hall" (Jackson, 1980, p. 273).

El contraste con el impacto de las propiedades cuánticas observadas posteriormente no puede ser mayor.

\section{El efegto Hall guántigo entero}

El nacimiento del efecto Hall cuántico puede fijarse muy precisamente. Fue la noche del 4 al 5 de febrero de 1980 alrededor de las 2 a.m. durante un experimento en el Laboratorio de Campo Magnético Intenso en Grenoble (von Klitzing, 2004, p. 1).

Ese dato, que parece menor, es relevante desde un punto de vista histórico. Aquí nace una corriente de investigaciones y de resultados que cambiaron completamente el perfil de las interpretaciones sobre las propiedades cuánticas de objetos y estados usualmente considerados fuera del alcance de esa rama de la física. Observando el contexto con cierto detalle, hay trabajos previos que presagian, de algún modo, esas novedades, aunque ello no es directo y la evaluación de su importancia es claramente a posteriori. En particular, es de mencionar un artículo de Ando (1974) sobre sistemas bidimensionales bajo la influencia de campos magnéticos. También es atendible el desarrollo de la física de materia condensada para esa época, particularmente en relación con las interacciones entre electrones y campos magnéticos. La física en dos dimensiones comenzaba a cobrar presencia protagónica, naturalmente acompañada de muy bajas temperaturas y campos magnéticos intensos. Adicionalmente, la tecnología de semiconductores ya mostraba fronteras de interés epistemológico. Los MOSFETs - los transistores de efecto de campo metal-óxido-semiconductor - son los grandes protagonistas tecnológicos en esse cambio conceptual.

Conviene hacer un comentario sobre el alcance de la física de dos dimensiones en ese ámbito cuántico. Como los niveles de energía son discretos, a muy bajas temperaturas los niveles más altos no son accesibles y, por ello, algunos tipos de movimien- 
tos no se pueden dar; esto es, se suprimen ciertos grados de libertad. Al establecer capas en un semiconductor de modo que el movimiento de los electrones en la tercera dimensión está cuantizado, se logra un ámbito sumamente preciso de dos dimensiones. Éste es un punto interesante para atender, sobre todo por las consecuencias representacionales y el estatus de las idealizaciones en torno de 2-D. La física de 2-D, en ese sector, cobra vida propia y sugiere un tratamiento similar al que reciben otras áreas en 3-D.

El aspecto novedoso de la investigación experimental de von Klitzing radica en que él estaba interesado en la caracterización del transporte electrónico en los transistores y en el deseo de mejorar la movilidad de esos dispositivos. Era importante encontrar y comprender los procesos de dispersión que dominan la movilidad mencionada. El foco estuvo puesto en capas finas en inter-fases especiales de silicio y compuestos de silicio. Para esos fines usó dispositivos Hall que le permitían mediciones directas del tensor de resistividad. Es de mencionar, para una adecuada captación del aporte de von Klitzing, que ya se conocía que los electrones forman un gas electrónico bidimensional cuando se acumulan en la superficie de un cristal de silicio como consecuencia de un voltaje positivo en el puente. Por último, el ambiente del laboratorio era especial. Las temperaturas que manejó eran del orden de $4 \mathrm{~K}$ y los campos magnéticos eran considerablemente intensos.

Aquí apareció la novedad sorprendente. En una gráfica que realizó, poniendo la resistencia Hall y la resistencia longitudinal de un mosfet de silicio a temperatura del helio líquido como una función de la tensión de puerta (gate voltage), se observaron mesetas (plateaux) en la resistencia Hall. Algo totalmente inesperado. Era conocido que el espectro de energía consiste en niveles de energía con hendeduras entre ellos. Otro aspecto relevante en los resultados de von Klitzing fue la estabilidad del fenómeno bajo mediciones de alta precisión, a pesar de las impurezas de los materiales y de las diferencias entre ellos. Los notables valores numéricos enteros obtenidos tuvieron una influencia directa sobre la metrología. Apareció una constante, conocida hoy como constante de von Klitzing, y sugirió, entre otras cosas, una conexión con otras constantes universales, como la constante de estructura fina. De allí el título del primer envío, "Realización de un estándar resistente baseado en constantes fundamentales". Como consecuencia de observaciones recibidas por los referís de la revista a la que se había enviado, éste cambió en "Nuevo método para la determinación de alta precisión de la constante de estructura fina baseado en la resistencia Hall cuantizada" (1980). Los otros autores de este trabajo, Dorda y Pepper, aportaron las muestras que usó von Klitzing. Él observó que la conductancia Hall sobre las mesetas era constante de muestra en muestra, y aproximadamente $n e^{2} / h$, donde $n$ es un entero determinado a una alta precisión. Aunque hubo sugerencias de que ese ámbito podría producir física intere- 
sante, parece que nadie se dio cuenta de la notable cuantización de la conductividad Hall. Dada la precisión alcanzada en la estabilidad de este fenómeno, el efecto Hall cuántico llegó a ser la base para el estándar de resistencia. Los medios especializados se hicieron eco de eso inmediatamente (BMS, 1981).

Lo más impactante de ese resultado es que la precisión de la cuantización no depende de la forma del material, ni de la forma considerada para definir sus regiones de contacto. Se suele decir que no depende de la geometría del objeto ni de las imperfecciones del mismo, naturalmente, dentro de ciertas escalas. Se ha llegado a afirmar aún que las imperfecciones son necesarias para el efecto. Algo realmente impensable $a$ priori, dada la precisión alcanzada y la dependencia directa del efecto cuantizado, de constantes universales como $e$ y .

Comparando el efecto clásico con el cuántico, hay razones para comprender un lapso de tiempo tan prolongado. El contexto experimental del trabajo de Hall involucraba temperaturas ambientales y campos magnéticos de baja intensidad, por lo tanto, la sensibilidad de las mediciones acusaba la influencia de numerosos factores perturbadores. Las bajas temperaturas están ampliando las fronteras de la física.

\section{El efegto Hall guántigo fragcionario}

Es ilustrativo introducir el tema con el siguiente comentario de Stormer.

Eso implica que muchos electrones, actuando en concierto, pueden crear nuevas partículas que tienen una carga menor que la carga de cualquier electrón individual (...). Pero las cargas fraccionarias son realmente bizarras. No solo ellas son más pequeñas, sino que son exactamente 1/3 o 1/5 o 1/7 etc. de una carga electrónica, dependiendo de las condiciones bajo las que ellas son preparadas. Y, sin embargo, nosotros sabemos con certeza que ninguno de esos electrones se ha partido en pedazos (1999, p. 875).

Los detalles del descubrimiento, comentados por los protagonistas, son dignos de atención detallada. Aquí sólo se mencionarán algunos. El descubrimiento de von Klitzing en 1980, despertó gran interés por los sistemas de electrones bidimensionales, y hasta 1982, los teóricos expresaron su confianza en la comprensión de por qué la conductancia Hall tenía que ser cuantizada en múltiplos enteros de $e^{2} / h$. Pero el descubrimiento ese año del efecto Hall cuántico fraccionario por Tsui, Stormer y Gossard (1982) llegó como una verdadera sorpresa. Por ello, varios artículos se refirieron a él como el efecto Hall cuántico anómalo. Según Stormer, su colega Tsui, al ver el resulta- 
do exclamó que estaban en presencia de quarks, pero claramente era sólo una broma, ya que ambos conocían las importantes diferencias entre fermiones y bosones, y la cuantización de la carga en fracciones debía ir por caminos diferentes. Naturalmente, en lo primero que uno piensa cuando estudia esos fenómenos es en esa analogía; incluso hay artículos sugerentes al respecto (cf. Greiter, 1997), pero las sutilezas teóricas sobre el alcance de las mismas quedan en manos de los especialistas y es claramente un terreno de investigación abierta (cf. Wilczek, 2006a). En cualquier caso, el efecto Hall cuántico fraccionario ha quedado adherido al comportamiento electrónico, pero al comportamiento colectivo de los mismos. Éste es quizás el aspecto más llamativo y enigmático.

En la base de la cuantización del efecto entero, hay un conjunto de densidades especiales de electrones donde se llenan exactamente $n$ niveles de Landau. Cuando una de esas densidades de carga se mueve a la velocidad de arrastre Hall, encontramos el efecto entero. Lo que se necesita para una comprensión similar del efecto fraccionario es algún mecanismo que seleccione fracciones de llenado racionales de la densidad de electrones del nivel de Landau. Fue inmediatamente apreciado que cualquier mecanismo para eso requiere interacciones entre los electrones. Una explicación hermosa y convincente fue dada por Laughlin. Su función de onda variacional combina la estadística de Fermi y la analiticidad de la función de onda del nivel de Landau más bajo, lo que permite mantener a los electrones aparte. Esa separación baja la energía de Coulomb y da a la función de onda una gran coincidencia con el estado fundamental. El estado de Laughlin alberga un conjunto de excitaciones de cuasi-partículas del tipo de vórtices topológicos interesantes, aunque es necesario decir que eso fue explotado posteriormente por otros autores que enfatizaron conexiones entre la física y la topología dentro del efecto (cf. Avron et al. 2003). Esos cuasi-agujeros y cuasi-partículas tienen cargas fraccionarias no-fluctuantes, lo que constituye en sí un fenómeno interesante, aunque es realmente tan interesante como eso la observación de que ellos se comportan bajo una estadística fraccionaria, esto es, algo entre los fermiones y los bosones (cf. Wilczek, 1990).

La función de onda de Laughlin es una construcción sumamente original, propuesta para el estado fundamental de un gas electrónico bidimensional, colocado en un campo magnético uniforme en un medio ambiente uniforme, cuando el factor de llenado del más bajo nivel de Landau es un entero positivo impar. Con ella intentó explicar el efecto cuántico Hall fraccionario, pero también sirvió para predecir exitosamente la existencia de otros estados adicionales, también con carga fraccionaria, más allá del valor $1 / 3$. Esos nuevos estados fueron posteriormente observados y, con ello, se consolidó la interpretación teórica del fenómeno.

La función de Laughlin, 


$$
\left\langle z_{1}, \ldots, z_{\mathrm{N}} \mid 0, L\right\rangle=\mathcal{N} \prod_{i<j}\left(z_{i}-z_{j}\right)^{2 \mathrm{n}+1} e^{-\frac{1}{4} \sum_{i=1}^{N}\left|z_{i}\right|^{2}}
$$

posee un conjunto de características propias que han sido considerablemente analizadas en trabajos posteriores. Por ejemplo, el estado $|0, L\rangle$ es holomórfico en cada coordenada electrónica y eso significa que es un estado de muchos cuerpos en el nivel más bajo de Landau. Además, para $n$ entero, $|0, L\rangle$ es anti-simétrico bajo el intercambio entre $z_{i} y z_{j}$, siendo válido el principio de Pauli. Por otra parte, los ceros múltiples, $\left(z_{i}-z_{j}\right)^{2 n+1}$, garantizan que la amplitud para que dos electrones estén próximos es muy pequeña. ${ }^{2}$

El aporte teórico de Laughlin es realmente impresionante, por su ansatz, por su concepción de la física en ese dominio, por su particular visión del rol de los modelos en la disciplina y por la contundencia de su enfoque al momento de evaluar los resultados de alta precisión. Lo más adecuado es aludir a algunas de sus reflexiones al respecto:

el efecto Hall cuántico es fascinante por una larga lista de razones, pero es importante, a mi modo de ver, principalmente por una: establece experimentalmente que ambos, las partículas llevando una fracción exacta de la carga del electrón e, y las poderosas fuerzas de gauge entre esas partículas, dos postulados centrales del modelo estándar de partículas elementales, pueden aparecer espontáneamente como fenómenos emergentes (Laughlin 1999, p. 873).

En ese sentido, es un defensor de la emergencia, mostrando una franca posición anti-reduccionista. Por otra parte, también vale citar su ponderación de los modelos en ese campo particular de la ciencia,

enfatizo esas cosas, porque existe una lamentable tendencia en física del estado sólido a igualar una comprensión de la naturaleza con una habilidad para construir un modelo, una actitud que algunas veces conduce a pasar por alto o interpretar mal el mayor principio organizador realmente responsable de un efecto. En el caso de los efectos cuánticos Hall enteros o fraccionarios, el punto esencial es la exactitud de la cuantización. Ninguna cantidad de modelización, hecha sobre alguna computadora existente o contemplada, explicará alguna vez esa exactitud por sí misma. Sólo un principio termodinámico puede hacerlo (...). Dados esos hechos, la más simple y más obvia explicación, en realidad la única concebi-

2 Para un desarrollo detallado, cf. Laughlin, 1983; para desarrollos posteriores, cf. Dyakonov, 2003; Murthy \& Shankar, 2003; Stone, 1992. 
ble, es que el nuevo estado es adiabáticamente deformable en algo físicamente similar a un nivel de Landau lleno, excepto con excitaciones cargadas de modo fraccionario (...) el estado Hall cuántico fraccionario es algo sin precedentes, un nuevo estado de la materia (Laughlin, 1999, p. 873).

Hay una curiosidad respecto de la construcción del científico que vale la pena mencionar. Stormer, un físico experimental y co-descubridor del efecto fraccionario, ha reiterado su admiración por la descripción de Laughlin y el alto poder explicativo y predictivo de la misma. Sin embargo, para Laughlin, eso fue producto en buena medida de la suerte que tuvieron los descubridores del fenómeno de encontrar primero la fracción 1/3 de la carga, ya que si hubieran encontrado alguna de las otras fracciones primero, probablemente no se habría logrado una adecuada comprensión ni representación tan fácilmente. Un buen guiño de la naturaleza. Pero ella también juega su juego de relativa autonomía. Los números impares también tuvieron su cota experimental en torno de los valores fraccionarios de la carga. Luego de un breve descanso en la ortodoxia asociada con ellos, apareció un valor de llenado de 5/2 que es consistente con una carga e/4, pero no con e/2 (cf. Dolev et al., 2008). Por esa razón, esa cuasipartícula fue imaginada como la mitad de un vórtice. Eso naturalmente obligó a repensar varias cuestiones del modelo teórico, pero también a ubicar en su lugar a los valores impares dentro del efecto fraccionario.

\section{DesarRollos POSTERIORES Y GONSIDERAGIONES FINALES}

Una de las consecuencias más llamativas, posteriores al trabajo de Laughlin, ha sido la participación de la topología matemática en ese dominio, como consecuencia de tratar de entender correctamente por qué se dan esos resultados experimentales tan precisos a pesar de las irregularidades e impurezas de los materiales. La conductancia Hall en la meseta tiene significado topológico, y aquí entran ciertos invariantes topológicos, a saber, los números de Chern. El número de Chern es topológico en el sentido de que es invariante ante pequeñas deformaciones del hamiltoniano. Pequeños cambios en el hamiltoniano resultan en pequeños cambios en la curvatura adiabática y, en consecuencia, se puede pensar en pequeños cambios en el número de Chern. El trabajo de Chern - Simons apareció en una revista de matemática en 1974, pero sus conexiones con la física teórica han mostrado múltiples facetas hasta la fecha, como son los abordajes de los invariantes Chern-Simons-Witten. Volviendo al efecto considerado aquí, desde un punto de vista físico, es llamativa la obstinación de los electrones para seguir ciertos derroteros y mantener la estabilidad de ciertos procesos, a pesar de las 
anomalías del medio en el que se encuentran. Se han ensayado varias maneras de describir las conexiones entre las impurezas y la topología. Un buen puente es el trabajo de Arovas et al. (1984), en el que desarrollan una idea de Schrieffer para el poliacetileno, y usan la fase de Berry y el teorema integral de Cauchy. En realidad, la fase de Berry es una buena conexión entre esos campos a través del transporte paralelo y la curvatura adiabática. Además, lo que encontraron esos investigadores fueron notables sutilezas sobre la estadística. En otro nivel de abstracción, usaron una construcción tipo ChernSimmons para modelos de electrones como vórtices de un campo de gauge ficticio. Eso sugiere un rostro profundo del efecto Hall cuántico que ha estimulado el desarrollo explosivo de muchas líneas de investigación en las últimas décadas. Una de ellas se está transformando en una industria prolífica, la investigación teórica sobre los aniones, que conectan diversos campos de modos realmente inusitados (cf. Avron et al., 2003; Stern, 2008; Wilczek, 2006b). Todo eso conduce a una reflexión filosófica, realizada principalmente por físicos, sobre la naturaleza de la carga y sus propiedades de cuantización. En ese sentido, complementa ciertos tratamientos del tema que provienen de las teorías cuánticas de campo. Las propiedades de la carga, entendida como una cantidad conservada, aditiva y discreta, están siendo re-contextualizadas (cf. Goldhaber, 2003).

Vale señalar que las redes conceptuales proliferan en esos dominios. Ciertos autores están señalando conexiones entre la superconductividad, la estadística y el efecto Hall cuántico, extendiendo el escenario a propiedades de nuevas entidades teóricas, como es el caso de los modelos de superconductividad de aniones y fermiones compuestos. Las propiedades matemáticas asociadas con esos modelos hablan con elocuencia y sugieren líneas de investigación que no eran pensadas hace algunos años, como las fases topológicas no abelianas. Éstas configuran sistemas extraños en los que un conjunto de excitaciones tipo-partículas del sistema, llamadas cuasi-partículas, tiene muchos estados con la misma energía. Si es el caso de un único estado, el orden topológico se dice que es abeliano (cf. Stern, 2010). Por citar una proyección extrema, la especulación en ese dominio ha llegado tan lejos como a la computación cuántica (cf. Nayak et al., 2008).

Como fue expresado arriba, hay otros fenómenos que han recibido el nombre de efecto Hall. El más significativo, por sus resultados en la actualidad, es el efecto Hall cuántico de espín. Se trata de un fenómeno que fue anticipado teóricamente, pero no en conductores, sino en ciertos aislantes muy delgados. Ese efecto involucra tanto electrones con espín hacia arriba conduciendo a lo largo de un borde del aislante, como electrones con espín hacia abajo conduciendo a lo largo del otro lado. A pesar de ser materiales aislantes en bulto, permiten la conducción en los bordes. Algo que diferencia a esos fenómenos de los anteriores es que los canales de transporte de corriente 
son posibles en ausencia de un campo magnético. El efecto Hall de espín señaló el comienzo de una nueva y fructífera corriente de investigación, los aislantes topológicos. Éstos, del mismo modo que en el efecto Hall de espín, tienen transportes separados de la carga, pero en cuatro canales. Esos canales se mueven hacia atrás y adelante para electrones con espín hacia arriba y hacia abajo, y también están protegidos topológicamente de impurezas y perturbaciones geométricas. Fueron predichos y recientemente observados. No obstante, la conexión con el efecto Hall clásico y cuántico es sólo parcial. Comparten la bi-dimensión y el efecto sobre los bordes, pero ya no comparten con los anteriores la presencia de un campo magnético como artífice del fenómeno. Gabe destacar que hay un componente tecnológico fundamental en ese dominio que está allanando el camino experimental de modo notable: el grafeno. En lo que respecta al efecto Hall, ese material ha permitido su obtención a temperaturas ordinarias, lo que está significando un avance notable en ciertas facetas experimentales del fenómeno (cf. Qi \& Zhang, 2011; Hasan \& Kane, 2010; Moore, 2010; Brüne et al., 2012).

\section{Conclusiones}

No hay aportes filosóficos destacables en este artículo. El mismo ha sido más bien un resultado de un tour de force orientado a un gigantesco y muy variado campo de investigación en una de las fronteras de la física cuántica aplicada. Se trata de dominios, por un lado, muy alejados de nuestras representaciones usuales y, por otro, muy cercanos, debido al aporte efectivo en ciencias de materiales. Esa composición configura un paisaje extraño, pero que es muy rico en sugerencias epistemológicas en torno de las bases teóricas y experimentales de la física contemporánea. Obviamente, sólo se han intentado explorar algunas facetas de ese paisaje. La cuantización de la carga es una pieza central de la física contemporánea. Pero los descubrimientos aludidos aquí no surgieron de especulaciones teóricas, ni de experimentos en la frontera de la física de altas energías. Han sido el producto de fenómenos concretos relativamente cercanos a nuestro mundo cotidiano. A partir de allí, se han construido expresiones matemáticas de gran poder explicativo y predictivo, como la función de onda variacional de Laughlin, de un modo similar a lo que aconteció con la superconductividad, a partir de la función de onda variacional construida por Bardeen, Cooper y Schrieffer para ese entorno. A eso hay que sumar el hecho de que los sistemas bidimensionales de electrones en fuertes campos magnéticos revelan una física de muchas partículas totalmente nueva, lo que refuerza la tesis de Anderson, “más es diferente”. Se ha mencionado arriba que Laughlin se ha transformado en un claro defensor del emergentismo. En cualquier caso, las conexiones teóricas han soltado sus riendas: solitones, computación cuántica, 
aniones, axiones, fermiones de Majorana etc. La especulación no presenta límites, y los resultados permiten albergar optimismo, como en el caso de esos últimos fermiones, muy particulares por cierto y que surgieron de un trabajo teórico de 1937 (cf. Mourik et al., 2012).

El efecto Hall se ha extendido hacia numerosos dominios de la física teórica. Von Klitzing (2004) hizo oportunamente una lista de conexiones teóricas muy variadas: con agujeros negros, quarks, teoría de cuerdas, el campo gravitacional, generalizaciones a 4 dimensiones. A pesar de los pocos años que tiene esa lista, se podrían añadir varias áreas adicionales, las que forman parte actualmente de la dinámica especulativa en torno del tema. Un ejemplo de ellas, la geometría no conmutativa, que ya forma parte de algunos textos sobre el efecto Hall, como es todo un capítulo de la segunda edición del libro de Ezawa (2008). Otro tópico que está usualmente presente en esas generalizaciones es la extensión a 4 dimensiones, bajo el objetivo canónico de unificar contextos teóricos. Si bien se sabe que ésta es una empresa muy difícil, cada tanto aparece algún trabajo que genera optimismo, aunque no siempre adecuadamente fundamentado (cf. Rodgers, 2001; Zhang-Hu, 2001).

Muchas preguntas pueden proponerse para generar una agenda programática en la investigación epistemológica sobre ese dominio. ¿Qué implicaciones tiene ese tema para una adecuada caracterización del reduccionismo? ¿Cuál es la relación entre las leyes micro y macro? ¿Qué lugar ocupan los "muchos objetos”? ¿Cuál es el umbral para "muchos"? ¿Se entiende adecuadamente el comportamiento colectivo de los electrones? ¿Aporta algo el enfoque de la complejidad a la investigación física, o es sólo un modo a posteriori de describir resultados no esperados desde una perspectiva compatible con el reduccionismo? ¿Qué podría significar una genuina ley descubierta que no puede ser obtenida desde leyes de un estrato inferior? ¿Son esas nuevas leyes autónomas o exhiben un comportamiento similar al de las leyes restringidas por fuertes condiciones iniciales? ¿Son esas condiciones iniciales sólo mundos posibles que configuran los campos de aplicación de las leyes "básicas"?

La pregunta que seleccionamos aquí es ésta: ¿pueden aportar algo esas leyes complejas para esclarecer mejor el significado y alcance de las leyes básicas? Una primera respuesta es sí. Sin embargo, es razonable suponer que mientras esa pregunta no sea adecuadamente respondida, no se tendrá un enfoque robusto sobre el estatus epistemológico de la complejidad. La principal interpretación que parece extraerse de los escritos de los científicos que se han transformado en voceros de la complejidad en el ámbito de la física es que las leyes de base no dicen todo. A partir de allí, elaboran toda una gama de posiciones frente a la novedad no prevista por esas leyes, en particular, la emergencia. Lo que parece salir reforzado de un análisis de efectos físicos, como el analizado en este artículo, es un constructivismo como el propuesto por Anderson 
(1972), pero ello no anula la posibilidad de que en el futuro esas novedades sean absorbidas por la ortodoxia reduccionista dominante en ese momento. En ese sentido, sería un nuevo ejemplo de la vieja tensión asociada con la dualidad de contextos: la búsqueda o descubrimiento, y la justificación. Lo que nadie duda es que la naturaleza sigue ofreciendo novedades, algunas anticipadas y otras no. Por ello, suena como propio de una visión muy estrecha el intento de cerrar el ámbito de una pregunta como la anterior para forzar una respuesta que lleva consigo los riesgos de ser demasiado a priori. En cualquier caso, la precisión de las mediciones ha sido aquí uno de los motores más significativos en esa dinámica, tanto teórica como experimental. Más aún, la historia del efecto analizado muestra que ciertas entidades "teóricas" no surgieron del escritorio, sino de resultados emergentes de las prácticas en los laboratorios. $\circledast$

\title{
Víctor RODRÍGuez
}

Profesor titular de la Escuela de Filosofía, Facultad de Filosofía y Humanidades, Universidad Nacional de Córdoba, Argentina. gauchovrr@gmail.com

\section{The quantum Hall effect and its contexts}

\begin{abstract}
In this paper we address several conceptual and historical aspects of the quantum Hall effect. We argue that this effect offers a variety of perspectives for philosophical reflection, from the generation of theoretical entities to the epistemology of experimentation. The exposition attempts to maintain a certain sensitivity to the historical dynamics around the issue, considering at the same time its metrological implications for specific quantum areas. Given the vast scientific literature on the subject, a cut is made in order to rescue some meaningful profiles of the phenomena associated with this effect.
\end{abstract}

KEYwords • Classical Hall effect. Quantum Hall effect. Quantum topology.

\section{REFERENGIAS BIBLIOGRÁFIGAS}

Ando, T. Theory of quantum transport in a two-dimensional electron system under magnetic fields. Journal of Physics Society, 37, p. 622-30, 1974 .

Anderson, P. W. More is different. Science, 177, 4.047, p. 393-6, 1972.

. Physics: the opening to complexity. Proceeding NationalAcademy of Science USA, 92, p. 6653-4, 1995 .

Arovas, D. et al. Fractional statistics and the quantum Hall effect. Physics Review Letters, 53, p. 722-3, 1984 . 


\section{El efecto Hall guántico y sus contextos}

Avron, E. et al. A topological look at the quantum Hall effect. Physics Today, 56, 8, p. 38-42, 2003.

Bms News. Search and discovery: quantized Hall effect yields $e^{2} / h$ to a part per million. Physics Today, 19 , p. 17-19, 1981.

BRÜNE C. et al. Spin polarization of the quantum spin Hall edge states. Nature Physics, 8, p. 4.85-90, 2012.

Chern, S. S. \& Simons, J. Characteristic forms and geometric invariants. Annals of Mathematics, 99, 1, p. $48-69,1974$.

Dolev, M. et al. Observation of a quarter of an electron charge at the $\mathrm{v}=5 / 2$ quantum Hall state. Nature, 452, p. $829-34,2008$.

Dyakonov, M. I. Twenty years since the discovery of the fractional quantum Hall effect: current state of the theory. In: VAGner, I. D.; Wyder, P. \& Maniv, T. (Ed.). Recent trends in theory of physical phenomena in high magnetic fields. Berlin: Springer, 2003. p. 75-88.

EzAwa, Z. F. Quantum Hall effects. 2 ed. Singapore: World Scientific, 2008.

Greiter, M., Quantum Hall quarks. Physica, E 1, p. 1-6, 1997.

Girvin, S. M. The quantum Hall effect: novel excitations and broken symmetries. Bloomington: Course 2, Dept. of Physics, Indiana University, 1999.

Goldhaber, A. S. Fractional charge definitions and conditions. Journal of Mathematical Physics, 44, 8, p. $3607^{-19}, 2003$.

HaLl, E. H. On a new action of the magnet on electric currents. American Journal of Mathematics, 2, p. $287-92,1879$.

Hasan, M. \& Kane, C. L. Topological insulators. Review of Modern Physics, 82, 4, p. 3०45 2010.

JACKson, J. D. Electrodinámica clásica. Madrid: Alhambra, 1980.

JAIN, J. K. The composite fermion: a quantum particle and its quantum fluids. Physics Today, 53, 4, p. 3945,2000 .

LaUghlin R. B. Anomalous quantum Hall effect: an incompressible quantum fluid with fractionally charged excitations. Physics Review Letters, 50, 18, p. 1395-8, 1983.

. Nobel Lecture: Fractional quantization. Review of Modern Physics, 71, 4, p. 863-74, 1999.

. A different universe. Reinventing physics from the bottom down. Cambridge: Basic Books, 2005.

Maxweld, J. C. A treatise on electricity and magnetism. Oxford: Clarendon Press, 1873. 2 v.

Moore, J. The birth of topological insulators. Nature, 464, p. 194-8, 2010.

Mourik, V. et al. Signatures of Majorana Fermions in hybrid superconductor-semiconductor nanowire devices. Science, 336, 6084, p. 1003-7, 2012.

Murthy G. \& Shankar, R. Hamiltonian theories of the fractional quantum Hall effect. Review of Modern Physics 75, 4, p. 1101-58, 2003.

NAYAK, G. et al. Non-abelian anyons and topological quantum computation. Review of Modern Physics, 80 , 3, p. 1083-159, 2008.

Panofsky, W. \& Phillips, M. Classical electricity and magnetism. Nova York: Dover, 1962.

QI X. L. \& Zhang S. C. Topological insulators and superconductors. Review Modern Physics, 83, p. $1057^{-}$ $110,2011$.

Rodgers, P. New paths to the ultimate theory. Physics World, 14, 12, p. 5, 2001.

Stern, A. Anyons and the quantum Hall effect. A pedagogical review. Annals of Physics, 323, p. 204-49, 2008.

. Non-abelian states of matter. Nature, 464, p. 187-93, 2010.

Stone, M. (Ed.). Quantum Hall effect. Singapore: World Scientific, 1992.

Stormer, H. L. Nobel lecture: the fractional quantum Hall effect. Review Modern Physics, 71, 4, p. 875-89, 1999.

Tsur, D. et al. Two-dimensional magnetotransport in the extreme quantum limit. Physics Review Letters, 48,22, p. $1559,1982$. 
VAGner, I. D.; Wyder, P. \& Maniv, T. (Ed.). Recent trends in theory of physical phenomena in high magnetic fields. Berlin: Springer, 2003.

Von KLitzing, K. 25 years of quantum hall effect (QHE). A personal view on the discovery, physics and applications of this quantum effect. Séminaire Poincaré, 2, p. 1-16, 2004.

Von Kuitzing, K. et. al. New method for high-accuracy determination of the fine-structure constant based on quantized Hall resistance. Physics Review Letters, 45, 6, p. 494, 1980.

Weinberg, S. Dreams of a final theory. Nova York: Pantheon Books, 1993.

Wilczer, F. (Ed.). Fractional statistics and Anyon superconductivity. Singapore: World Scientific, 1990.

Wilczer, F. Some basic aspects of fractional quantum numbers. Fantastic Realities, 49, p. 359-83. 2006a. From electronics to anyonics. Physics World, 19, 1, p. 22-3, 2006b.

Zhang S. \& Hu, J. A four dimensional generalization of the quantum Hall effect. Science, 294, p. 823, 2001. 